\title{
High-order plate finite elements for smart structure analysis
}

\author{
O. Polit*, M. D’Ottavio, P. Vidal \\ Laboratoire Energétique, Mécanique, Electromagnétisme, Université Paris Ouest Nanterre-La Défense, 50 rue de Sèvres, 92410 Ville d'Avray, France
}

\section{A B S T R A C T}

This paper presents new finite elements for plate analysis of smart composite structures. Based on the sinus model, Murakami's Zig-Zag functions are introduced in the three directions, improving the accuracy for multilayered modeling. The transverse normal stress is included allowing use of the three-dimensional constitutive law. Three different eight-node finite elements are developed using $\mathrm{C}^{0}$ approximations, each with a different number of unknown functions: 9, 11 or 12 . For the piezoelectric approximation, a layer wise description is used with a cubic variation in the thickness of each layer while the potential is assumed to be constant on each elementary domain for the in-plane variation. These finite elements aims at modeling both thin and thick plates without any pathologies of the classical plate finite elements (shear and Poisson or thickness locking, spurious modes, etc.). This family is evaluated on classical piezoelectric problems of the literature and special emphasis is pointed towards the introduction of equipotential conditions.

\section{Introduction}

Research and development concerning high-performance structures are very intense since some decades. Structural health monitoring, active vibration damping, and energy harvesting are some examples of possible applications of a multifunctional structural component. Piezoelectric materials permit to convert mechanical and electrical energy at frequency ranges that are most interesting for technical applications such as vibration damping and rapid shape adaptation [1]. Development of theoretical and numerical models for this kind of structures is very important and active. For this purpose and in the framework of two-dimensional plate/ shell models, different choices can be made for the mechanical approximation and the following classification is classically admitted for the variation in the thickness direction: (i) Equivalent Single Layer (ESL) models, in which the number of unknowns is independent of the layer number; (ii) Layer-Wise (LW) descriptions, for which the number of unknowns and, thus, the computational cost increases with the number of layers. While most developments employ an ESL description for the mechanical behavior, and particularly the First order Shear Deformation Theory (FSDT), a LayerWise description is necessary for the piezoelectric approximation to impose electric boundary conditions at each piezoelectric layer interfaces, i.e., the electrodes, within the stack. Inside each piezo-

\footnotetext{
* Corresponding author.

E-mail address: olivier.polit@u-paris10.fr (O. Polit).
}

electric layer, the electric potential can be linear, quadratic or higher and a comparison has been proposed in [2].

A review of different approaches is available in $[3,4]$ and in the framework of the Carrera Unified Formulation (CUF) in [5]. For the FE approximations, a recent review limited to shell models is also given in [6].

The limitation of the FSDT model is related to the constant transverse displacement hypothesis, inducing no thickness change and the use of the reduced 2D constitutive law. The use of the full $3 \mathrm{D}$ constitutive law is an important feature for a consistent representation of complex physical interactions like multi-field coupling. Furthermore, accurate modeling of thick structures needs the transverse normal stress and the 3D constitutive law.

Therefore, a high-order model is chosen with sinus function for the in-plane displacements and quadratic assumption along the thickness of the transverse deflection. Thus, the 3D constitutive law is retained and a parabolic distribution of the transverse shear strains and a non linear variation of the transverse normal strain are recovered. In order to introduce transverse strain discontinuities required to fulfill the interlaminar equilibrium, Murakami's Zig-Zag function (MZZF) [7] is superimposed to the high-order ESL kinematics for the 3 displacement components. Note that MZZF does not depend on the constitutive coefficients and is, hence, attractively simple in conjunction with three-dimensional constitutive laws including multi-field coupling. Based on this kinematics, an 8-node plate finite element (FE) is proposed, free of numerical illness such as transverse shear and Poisson lockings, oscillation and spurious mechanics [8]. The approximation of the 
electric potential must be able to model piezoelectric patches, and a constant value is considered on each elementary domain while a cubic variation in each layer is used, based on the polynomial expansion given in $[9,10]$.

The paper is organized as follows: Section 2 describes the plate problem and the FE approximations are given in Section 3. The resulting FE are validated in Section 4 by referring to well-known linear static piezoelectric and composite plate problems. Finally, Section 5 summarizes the main findings.

\section{Description of the plate problem}

\subsection{Governing equations}

Let us consider a plate occupying the domain $\mathcal{V}=\Omega \times\left[-\frac{e}{2} \leqslant z \leqslant \frac{e}{2}\right]$ in a Cartesian coordinate system $\left(x_{1}, x_{2}, x_{3}=z\right)$. The plate is defined by an arbitrary surface $\Omega$ in the $\left(x_{1}, x_{2}\right)$ plane, located at the midplane for $z=0$, and by a constant thickness $e$.

The displacement is denoted $\vec{u}\left(x_{1}, x_{2}, z\right)$ and the electric potential is $\phi\left(x_{1}, x_{2}, z\right) . \varepsilon_{i j}\left(x_{1}, x_{2}, z\right)$ and $\vec{E}\left(x_{1}, x_{2}, z\right)$ are the strain tensor components and the electric field vector, respectively, deduced from primal variables by the geometric relations. Furthermore, $\sigma_{i j}\left(x_{1}, x_{2}, z\right)$ and $\vec{D}\left(x_{1}, x_{2}, z\right)$ are the conjugated fluxes (stress tensor components and dielectric displacement vector, respectively) obtained from the constitutive equations given in the next subsection.

\subsubsection{Constitutive relation}

The 3D constitutive equation for a linear piezoelectric material is given by the following set of coupled equations [11] for a layer $(k)$ :

$$
\begin{aligned}
& {\left[\sigma^{(k)}\right]=\left[C^{(k)}\right]\left[\varepsilon^{(k)}\right]-\left[e^{(k)}\right]^{T}\left[E^{(k)}\right]} \\
& {\left[D^{(k)}\right]=\left[e^{(k)}\right]\left[\varepsilon^{(k)}\right]+\left[\epsilon^{(k)}\right]\left[E^{(k)}\right]}
\end{aligned}
$$

where we denote by $[C]$ the matrix of elastic stiffness coefficients taken at constant electric field, by $[e]$ the matrix of piezoelectric stress coefficients and by $[\epsilon]$ the matrix of electric permittivity coefficients taken at constant strain. The explicit form of these matrices can be found in [6] for an orthotropic piezoelectric layer polarized along the thickness direction $z$. Eq. (1a) expresses the piezoelectric converse effect for actuator applications, whereas Eq. (1b) represents the piezoelectric direct effect which is exploited in sensor applications. Note that the constitutive law is expressed in the local reference frame associated to each layer.

\subsubsection{The weak form of the boundary value problem}

The classical piezoelectric variational formulation of [12] is employed in which the primary field variables are the "generalized displacements", i.e., the displacement field and the electrostatic potential. Using a matrix notation and for admissible virtual displacements $\vec{u}^{*}$ and electric potential $\phi^{*}$ (virtual quantities are denoted by an asterisk), the variational principle is given by:

$$
\begin{aligned}
\int_{\mathcal{V}} \rho\left[u^{*}\right]^{T}[\ddot{u}] d \mathcal{V}= & -\int_{\mathcal{V}}\left[\varepsilon\left(u^{*}\right)\right]^{T}[\sigma(u, \phi)] d \mathcal{V}+\int_{\mathcal{V}}\left[u^{*}\right]^{T}[f] d \mathcal{V} \\
& +\int_{\partial \mathcal{V}_{F}}\left[u^{*}\right]^{T}[F] d \partial \mathcal{V}+\int_{\mathcal{V}}\left[E\left(\phi^{*}\right)\right]^{T}[D(u, \phi)] d \mathcal{V} \\
& -\int_{\mathcal{V}} q \phi^{*} d \mathcal{V}-\int_{\partial \mathcal{V}_{\mathcal{Q}}} Q \phi^{*} d \partial \mathcal{V}
\end{aligned}
$$

where $[f]$ is the body force vector, $[F]$ the surface force vector applied on $\partial \mathcal{V}_{F}, q$ the volume charge density, $Q$ the surface charge density supplied on $\partial C_{q}$ and $\rho$ is the mass density. Finally, $\varepsilon\left(u^{*}\right)$ and $E\left(\phi^{*}\right)$ are the virtual strain and virtual electric field that satisfy the compatibility gradient equations. In the remainder of this article we will refer only to static problems, for which the left-hand side term is set to zero. Furthermore, body forces and volume charge densities will be discarded $([f]=[0] ; q=0)$.

\subsection{The mechanical part}

\subsubsection{The displacement field}

Based on the sinus model, see [13], a new plate model which takes into account the transverse normal stress is presented in this section. This extension is based on following developments:

- various models for beams, plates and shells based on the refined sinus theory, see [13-20];

- our previous paper on a 7 parameter model for thermomechanical analysis [8].

In the framework of ESL approach, the kinematics of our model is assumed to have the following particular form

$\left\{\begin{array}{l}U_{1}\left(x_{\alpha}, z\right)=u_{1}^{0}\left(x_{\alpha}\right)+z u_{1}^{1}\left(x_{\alpha}\right)+f(z) u_{1}^{f}\left(x_{\alpha}\right) \\ U_{2}\left(x_{\alpha}, z\right)=u_{2}^{0}\left(x_{\alpha}\right)+z u_{2}^{1}\left(x_{\alpha}\right)+f(z) u_{2}^{f}\left(x_{\alpha}\right) \\ U_{3}\left(x_{\alpha}, z\right)=u_{3}^{0}\left(x_{\alpha}\right)+z u_{3}^{1}\left(x_{\alpha}\right)+z^{2} u_{3}^{2}\left(x_{\alpha}\right)\end{array}\right.$

where $\alpha \in\{1,2\}$ and $i \in\{1,2,3\}$. In Eq. (3), the superscript is associated to the expansion order in $z$ while the subscript is related to the component of the displacement. Thus, $u_{i}^{0}$ are the displacements of a point of the reference surface while $\left(u_{\alpha}^{1}, u_{\alpha}^{f}\right)$ are measures for rotations of the normal transverse fiber about the axis $\left(0, x_{\alpha}\right)$. The functions $u_{3}^{\alpha}$ permit to have a non-constant deflection for the transverse fiber and allow to have non zero transverse normal stretch. Furthermore, the quadratic assumption for the transverse displacement avoids the occurrence of Poisson (or thickness) locking, see [8].

In the context of the sinus model, we have

$f(z)=\frac{e}{\pi} \sin \frac{\pi z}{e}$

It must be noticed that the classical homogeneous sinus model [13] can be recovered from Eq. (3) assuming $u_{\alpha}^{1}=-u_{3, \alpha}^{0}$, and neglecting the unknown functions $u_{3}^{\alpha}$.

The choice of the sinus function can be justified from the threedimensional point of view, using the work [21]. As it can be seen in [22], a sinus term appears in the solution of the shear equation (see Eq. (7) in [22]). Therefore, the kinematics proposed can be seen as an approximation of the exact three-dimensional solution. Furthermore, the sinus function has an infinite radius of convergence and its Taylor expansion includes not only the third order terms but all the odd terms.

\subsubsection{The Murakami's Zig-Zag terms}

In order to evaluate the influence of Zig-Zag terms [7] in a highorder ESL model, the following displacement per layer $(k)$ are added to Eq. (3):

$\left\{\begin{array}{l}U_{1}^{(k)}\left(x_{\alpha}, z\right)=Z^{(k)}(z) u_{1}^{z}\left(x_{\alpha}\right) \\ U_{2}^{(k)}\left(x_{\alpha}, z\right)=Z^{(k)}(z) u_{2}^{z}\left(x_{\alpha}\right) \\ U_{3}^{(k)}\left(x_{\alpha}, z\right)=Z^{(k)}(z) u_{3}^{z}\left(x_{\alpha}\right)\end{array}\right.$

with

$Z^{(k)}(z)=(-1)^{k} \zeta_{k}(z)$ and $\zeta_{k}(z)=\frac{2}{e_{k}}\left(z-\frac{1}{2}\left(z_{k}+z_{k+1}\right)\right)$ 
where $e_{k}$ is the thickness of the $k$ th layer while $\left(z_{k}, z_{k+1}\right)$ are the bottom and top coordinates of this layer. It is obvious that $Z^{(k)}(z)$ is a piecewise linear function with bi-unit amplitude for all the layers as we have $\zeta_{k}(z) \in[-1,1]$. Note that, despite the $Z^{(k)}(z)$ function depends on the layer index inside the stack, the amplitudes $u_{i}^{z}$ are unique for the whole laminate, i.e., the ESL framework is still preserved.

\subsubsection{The strain field}

The strain field in each layer $(k)$ is easily deduced from Eqs. (3) and (5) and we have

$$
\left\{\begin{array}{l}
\varepsilon_{11}^{(k)}=u_{1,1}^{0}+z u_{1,1}^{1}+f(z) u_{1,1}^{f}+Z^{(k)}(z) u_{1,1}^{z} \\
\varepsilon_{22}^{(k)}=u_{2,2}^{0}+z u_{2,2}^{1}+f(z) u_{2,2}^{f}+Z^{(k)}(z) u_{2,2}^{z} \\
\varepsilon_{33}^{(k)}=u_{3}^{1}+2 z u_{3}^{2}+Z^{(k) \prime}(z) u_{3}^{z} \\
\gamma_{23}^{(k)}=\gamma_{23}^{0}+f^{\prime}(z) u_{2}^{f}+z u_{3,2}^{1}+z^{2} u_{3,2}^{2}+Z^{(k)}(z) u_{3,2}^{z}+Z^{(k) \prime}(z) u_{2}^{z} \\
\gamma_{13}^{(k)}=\gamma_{13}^{0}+f^{\prime}(z) u_{1}^{f}+z u_{3,1}^{1}+z^{2} u_{3,1}^{2}+Z^{(k)}(z) u_{3,1}^{z}+Z^{(k) \prime}(z) u_{1}^{z} \\
\gamma_{12}^{(k)}=u_{1,2}^{0}+u_{2,1}^{0}+z\left(u_{1,2}^{1}+u_{2,1}^{1}\right)+f(z)\left(u_{1,2}^{f}+u_{2,1}^{f}\right)+Z^{(k)}(z)\left(u_{1,2}^{z}+u_{2,1}^{z}\right)
\end{array}\right.
$$

In Eq. (7), the functions $\gamma_{\alpha 3}^{0}$ are introduced in order to control the transverse shear locking as in [8]. These terms are constant with respect to the thickness coordinate $z$ and have the following expressions

$$
\left\{\begin{array}{l}
\gamma_{23}^{0}=u_{3,2}^{0}+u_{2}^{1} \\
\gamma_{13}^{0}=u_{3,1}^{0}+u_{1}^{1}
\end{array}\right.
$$

The following matrix notation for the strain $[\varepsilon]$ is used for its introduction in Eqs. (1) and (2),

$$
[\varepsilon]=\left[\begin{array}{llllll}
\varepsilon_{11} & \varepsilon_{22} & \varepsilon_{33} & \gamma_{23} & \gamma_{13} & \gamma_{12}
\end{array}\right]^{T}=\left[F_{\varepsilon}\right]\left[\mathcal{E}_{\varepsilon}\right]
$$

where the generalized strain vector $\left[\mathcal{E}_{\varepsilon}\right]$ is given by: and $\left[C s t_{\phi}^{(k)}\right]$ is a $(4 \times 3)$ matrix containing constant coefficients. The electric field vector in each layer $\left[E^{(k)}\right]$ is then obtained as:

$$
\left[E^{(k)}\right]=\left[\begin{array}{c}
0 \\
0 \\
-\phi_{, 3}^{(k)}
\end{array}\right]=\left[F_{E}\right]\left[C s t_{E}^{(k)}\right]\left[q_{\phi}^{e(k)}\right] \text { with }\left[F_{E}\right]=\left[\begin{array}{lll}
1 & z & z^{2}
\end{array}\right]
$$

where $\left[C s t_{E}^{(k)}\right]$ is a $(3 \times 3)$ matrix. So, the adopted approximation yields a quadratic transverse electric field across the thickness of each layer.

\subsection{The bi-dimensional weak form}

Starting from the weak form Eq. (2), integrations in the thickness direction for each layer $(k)$ are conducted in order to define the bi-dimensional problem on $\Omega$. Introducing Eq. (1) and using Eqs. (9) and (13), the following expression is deduced

$$
\begin{aligned}
\int_{\Omega} & {\left[\mathcal{E}_{\varepsilon}^{*}\right]^{T}\left[k_{u u}\right]\left[\mathcal{E}_{\varepsilon}\right] d \Omega-\int_{\Omega}\left[\mathcal{E}_{\varepsilon}^{*}\right]^{T}\left[k_{u \phi}\right]\left[C s t_{E}\right]\left[q_{\phi}^{e}\right] d \Omega } \\
& -\int_{\Omega}\left[q_{\phi}^{e *}\right]^{T}\left[C s t_{E}\right]^{T}\left[k_{\phi u}\right]\left[\mathcal{E}_{\varepsilon}\right] d \Omega \\
& -\int_{\Omega}\left[q_{\phi}^{e *}\right]^{T}\left[C s t_{E}\right]^{T}\left[k_{\phi \phi}\right]\left[C s t_{E}\right]\left[q_{\phi}^{e}\right] d \Omega \\
& =P_{\text {ext }}(V)
\end{aligned}
$$

In Eq. (14), the integration of the constitutive equations with respect to the thickness has been done for each layer $(k)$ in the following matrices:

$\left[\mathcal{E}_{\varepsilon}\right]^{T}=\left[\begin{array}{llllllllllllllllllllllllllllllllll}u_{1,1}^{0} & u_{1,2}^{0} & u_{2,1}^{0} & u_{2,2}^{0} \mid & u_{1,1}^{1} & u_{1,2}^{1} & u_{2,1}^{1} & u_{2,2}^{1} \mid \gamma_{23}^{0} & \gamma_{13}^{0} & u_{1}^{f} & u_{1,1}^{f} & u_{1,2}^{f} & u_{2}^{f} & u_{2,1}^{f} & u_{2,2}^{f} \mid u_{3}^{1} & u_{3,1}^{1} & u_{3,2}^{1} \mid & u_{3}^{2} & u_{3,1}^{2} & u_{3,2}^{2} & u_{1}^{z} & u_{1,1}^{z} & u_{1,2}^{z} & u_{2}^{z} & u_{2,1}^{z} & u_{2,2}^{z} & u_{3}^{z} & u_{3,1}^{z} & u_{3,2}^{z}\end{array}\right]$

The matrix $\left[F_{\varepsilon}\right]$ can easily be deduced from Eq. (7) and is not detailed here.

\subsection{The electric part}

On each elementary domain $\Omega_{e}$, the electric potential is assumed to be constant. Therefore, no variation with respect to $x_{\alpha}$ is considered. A cubic Layer-Wise (LW) description is used across the thickness, according to the approximation introduced in [9] and used in [10]: In each layer $(k)$, the electric potential distribution is described by the normal electric field components at the bottom and top surfaces, denoted $E_{3 b}$ and $E_{3 t}$, respectively, and the potential difference $\Delta \phi$ between top and bottom surface. Using these dofs, the approximation of the electric potential can be written as:

$$
\left[\phi^{(k)}\right]=\left[F_{\phi}\right]\left[C s t_{\phi}^{(k)}\right]\left[q_{\phi}^{e(k)}\right]
$$

where the following definitions have been introduced:

$$
\left[F_{\phi}\right]=\left[\begin{array}{llll}
1 & z & z^{2} & z^{3}
\end{array}\right] ; \quad\left[\begin{array}{ll}
q_{\phi}^{e(k)}
\end{array}\right]^{T}=\left[\begin{array}{lll}
E_{3 b}^{(k)} & \Delta \phi^{(k)} & E_{3 t}^{(k)}
\end{array}\right]
$$

$$
\begin{aligned}
& {\left[k_{u u}^{(k)}\right]=\int_{z_{k}}^{z_{k+1}}\left[F_{\varepsilon}\right]^{T}\left[C^{(k)}\right]\left[F_{\varepsilon}\right] d z} \\
& {\left[k_{u \phi}^{(k)}\right]=\int_{z_{k}}^{z_{k+1}}\left[F_{\varepsilon}\right]^{T}\left[e^{(k)}\right]^{T}\left[F_{E}\right] d z \text { and }\left[k_{\phi u}^{(k)}\right]=\left[k_{u \phi}^{(k)}\right]^{T}} \\
& {\left[k_{\phi \phi}^{(k)}\right]=\int_{z_{k}}^{z_{k+1}}\left[F_{E}\right]^{T}\left[\epsilon^{(k)}\right]^{T}\left[F_{E}\right] d z}
\end{aligned}
$$

In this expression, the layer $(k)$ is indicated for the matrices involving the LW approach used for the electric potential and the Zig-Zag functions for the displacement. Therefore, a summation must be done for all the layers during an assembling process to obtain $\left[k_{u u}\right],\left[k_{u \phi}\right],\left[k_{\phi \phi}\right]$ of Eq. (14).

\section{The finite element system}

\subsection{Finite element approximations}

Eq. (14) is a good starting point for introducing the FE approximations, whose details shall be here omitted for the sake of brevity and can be found elsewhere [6,8]. The eight-node quadrilateral finite element is used and classical FE approximation is used for 


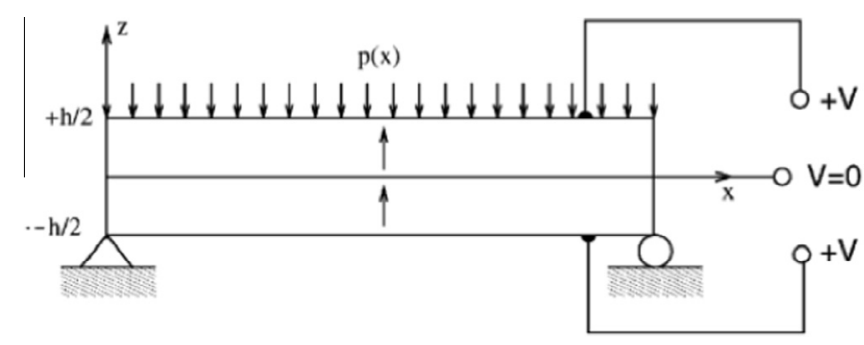

Fig. 1. Configurations of the piezoelectric bimorph with parallel poling direction along $z$-Sensor: $p(x)=p_{0}, V=0$; Actuator: $V=\Delta \phi_{0}, p(x)=0$.

the geometry. A special treatment is used to control the transverse shear locking by using a dedicated interpolation for $\gamma_{\alpha 3}^{0}$ according to the methodology presented in [8]. Note that since the electric potential is assumed to be constant on each elementary domain, there is no need to introduce any FE approximation for this field.

\subsection{The system to be solved}

The elementary matrices are deduced from the bi-dimensional weak form given in Eq. (14). Assembling each elementary contribution in the global reference frame, the following discrete form of the coupled piezoelectric system is obtained:

$$
\left[\begin{array}{cc}
{\left[K_{u u}\right]} & {\left[K_{u \phi}\right]} \\
{\left[K_{u \phi}\right]^{T}} & {\left[K_{\phi \phi}\right]}
\end{array}\right]\left[\begin{array}{l}
{\left[q_{u}\right]} \\
{\left[q_{\phi}\right]}
\end{array}\right]=\left[\begin{array}{c}
{\left[L_{u}\right]} \\
{\left[L_{\phi}\right]}
\end{array}\right]
$$

where $\left[K_{u u}\right],\left[K_{\phi \phi}\right]$ and $\left[K_{u \phi}\right]$ are the global stiffness, dielectric and piezoelectric matrices of the plate, respectively. The mechanical dofs are in the vector $\left[q_{u}\right]$, while the electrical dofs are in the vector $\left[q_{\phi}\right]$ and we have at the elementary level
Table 2

Parallel bimorph; $S=10$; mesh convergence study.

\begin{tabular}{lllllll}
\hline Mesh & Mech dof & Elec dof & $\bar{U}_{3}$ & $\bar{\sigma}_{11}$ & $\bar{\phi}$ & $\bar{D}_{3}$ \\
\hline \multicolumn{2}{l}{ Actuator configuration } & & & & & \\
$2 \times 2$ & 143 & 24 & 118.82 & 1.22 & 0.53 & -22.01 \\
$4 \times 2$ & 271 & 48 & 118.83 & 1.22 & 0.53 & -22.01 \\
$8 \times 2$ & 527 & 96 & 118.86 & 1.22 & 0.53 & -22.01 \\
$16 \times 2$ & 1039 & 192 & 118.89 & 1.22 & 0.53 & -22.01 \\
$32 \times 2$ & 2063 & 384 & 118.90 & 1.22 & 0.53 & -22.01 \\
Ansys & & & 118.37 & 1.22 & 0.50 & -21.96 \\
Sensor configuration & & & & & \\
$2 \times 2$ & 143 & 24 & 2360.1 & 80.91 & 0.69 & -52.37 \\
$4 \times 2$ & 271 & 48 & 2360.3 & 79.24 & 0.74 & -55.75 \\
$8 \times 2$ & 527 & 96 & 2362.3 & 78.80 & 0.75 & -56.83 \\
$16 \times 2$ & 1039 & 192 & 2366.7 & 78.68 & 0.75 & -57.08 \\
$32 \times 2$ & 2063 & 384 & 2370.3 & 78.64 & 0.75 & -57.14 \\
Ansys & & & 2348.1 & 78.53 & 0.77 & -52.28 \\
\hline
\end{tabular}

$\left[q_{u}^{e}\right]=\left[\begin{array}{lllllllllllllll}u_{1}^{0} & u_{2}^{0} & u_{3}^{0} & u_{1}^{1} & u_{2}^{1} & u_{3}^{1} & u_{1}^{f} & u_{2}^{f} & u_{3}^{2} & \left.\mid \begin{array}{lllll}z & u_{2}^{z} & u_{3}^{z}\end{array}\right)_{i=1,8}\end{array}\right]$

$$
\left.\left[q_{\phi}^{e}\right]=\left[\begin{array}{lll}
E_{3 b}^{(k)} & \Delta \phi^{(k)} & E_{3 t}^{(k)}
\end{array}\right)_{k=1, N l}\right]
$$

with $\mathrm{Nl}$ the number of layers. From the mechanical point of view, the Zig-Zag dofs can be activated or not and comparisons will be presented in the next section dedicated to numerical evaluations using no Zig-Zag dof (P9), using only in-plane Zig-Zag dof $u_{\alpha}^{z}$ (P9Z) and with all the Zig-Zag dof $u_{i}^{z}$ (P9ZZ). Finally, the three models use 9,11 or 12 kinematical unknown functions and the associated $\mathrm{FE}$ has 72, 88, 96 mechanical dofs per element, respectively.

Since the electric potential is assumed constant on each FE, the assembly involves only the approximation along the thickness. A piezoelectric patch comprising several FEs can be defined through the imposition of the equipotential condition between electrodes:

Table 1

\begin{tabular}{|c|c|c|c|c|c|c|c|c|c|}
\hline Prop. & PZT-4 & PZT-5A & Skin & Core & Prop. & PZT-4 & PZT-5A & Skin & Core \\
\hline$E_{1}[\mathrm{GPa}]$ & 81.24 & 61.0 & 172.5 & 0.276 & $e_{15}\left[\mathrm{C} / \mathrm{m}^{2}\right]$ & 12.7 & 12.3 & 0 & 0 \\
\hline$E_{2}[\mathrm{GPa}]$ & 81.24 & 61.0 & 6.9 & 0.276 & $e_{24}\left[\mathrm{C} / \mathrm{m}^{2}\right]$ & 12.7 & 12.3 & 0 & 0 \\
\hline$E_{3}[\mathrm{GPa}]$ & 64.07 & 53.2 & 6.9 & 3.45 & $e_{31}\left[\mathrm{C} / \mathrm{m}^{2}\right]$ & -5.2 & -7.2 & 0 & 0 \\
\hline$v_{23}$ & 0.43 & 0.38 & 0.25 & 0.02 & $e_{32}\left[\mathrm{C} / \mathrm{m}^{2}\right]$ & -5.2 & -7.2 & 0 & 0 \\
\hline$v_{13}$ & 0.43 & 0.38 & 0.25 & 0.02 & $e_{33}\left[\mathrm{C} / \mathrm{m}^{2}\right]$ & 15.1 & 15.1 & 0 & 0 \\
\hline$v_{12}$ & 0.33 & 0.35 & 0.25 & 0.25 & $\epsilon_{11}[\mathrm{nF}]$ & 13.06 & 15.3 & $\epsilon_{0}$ & $\epsilon_{0}$ \\
\hline$G_{23}[\mathrm{GPa}]$ & 25.6 & 21.1 & 1.38 & .414 & $\epsilon_{22}[\mathrm{nF}]$ & 13.06 & 15.3 & $\epsilon_{0}$ & $\epsilon_{0}$ \\
\hline$G_{13}[\mathrm{GPa}]$ & 25.6 & 21.1 & 3.45 & .414 & $\epsilon_{33}[\mathrm{nF}]$ & 11.51 & 15.0 & $\epsilon_{0}$ & $\epsilon_{0}$ \\
\hline$G_{12}[\mathrm{GPa}]$ & 30.6 & 22.6 & 3.45 & 0.1104 & & & & & \\
\hline
\end{tabular}

Material properties employed in the considered problems.

Table 3

\begin{tabular}{|c|c|c|c|c|c|c|c|c|}
\hline Model & Mech dof & Elec dof & $\bar{U}_{3}$ & $\bar{\sigma}_{11}$ & {$\left[\left[\bar{\sigma}_{11}\right]\right]_{0}$} & $\bar{\phi}$ & $\bar{D}_{3}$ & {$\left[\left[\bar{D}_{3}\right]\right]_{0}$} \\
\hline \multicolumn{9}{|c|}{ Actuator configuration } \\
\hline Ansys & & & 118.37 & 1.22 & 4.87 & 0.500 & -21.96 & 43.92 \\
\hline P9ZZ & 527 & 96 & 118.86 & 1.22 & 4.88 & 0.534 & -22.01 & 44.01 \\
\hline P9ZZ w/o Du $u_{3}^{2}$ & 527 & 96 & 118.72 & 1.22 & 4.88 & 0.534 & -22.01 & 44.01 \\
\hline P9Z & 458 & 96 & 112.66 & 0.47 & 1.90 & 0.567 & -20.98 & 41.97 \\
\hline P9 & 394 & 96 & 112.66 & 0.47 & 1.90 & 0.567 & -20.98 & 41.97 \\
\hline FSDT & 394 & 96 & 118.16 & 1.22 & 4.88 & 0.534 & -22.00 & 44.00 \\
\hline \multicolumn{9}{|c|}{ Sensor configuration } \\
\hline Ansys & & & 2348.1 & 78.53 & 13.19 & 0.77 & -52.28 & 104.53 \\
\hline P9ZZ & 527 & 96 & 2362.3 & 78.80 & 12.61 & 0.75 & -56.83 & 116.16 \\
\hline P9ZZ w/o Du $u_{3}^{2}$ & 527 & 96 & 2350.4 & 78.92 & 12.97 & 0.74 & -56.74 & 115.92 \\
\hline $\mathrm{P9Z}$ & 458 & 96 & 2345.5 & 76.92 & 5.00 & 0.84 & -54.23 & 110.74 \\
\hline P9 & 394 & 96 & 2345.5 & 76.92 & 5.00 & 0.84 & -54.23 & 110.74 \\
\hline FSDT & 394 & 96 & 2351.6 & 78.45 & 13.03 & 0.76 & -58.77 & 117.54 \\
\hline
\end{tabular}

Parallel bimorph in cylindrical bending $(S=10 ; 8 \times 2$ mesh): influence of the Zig-Zag terms. 

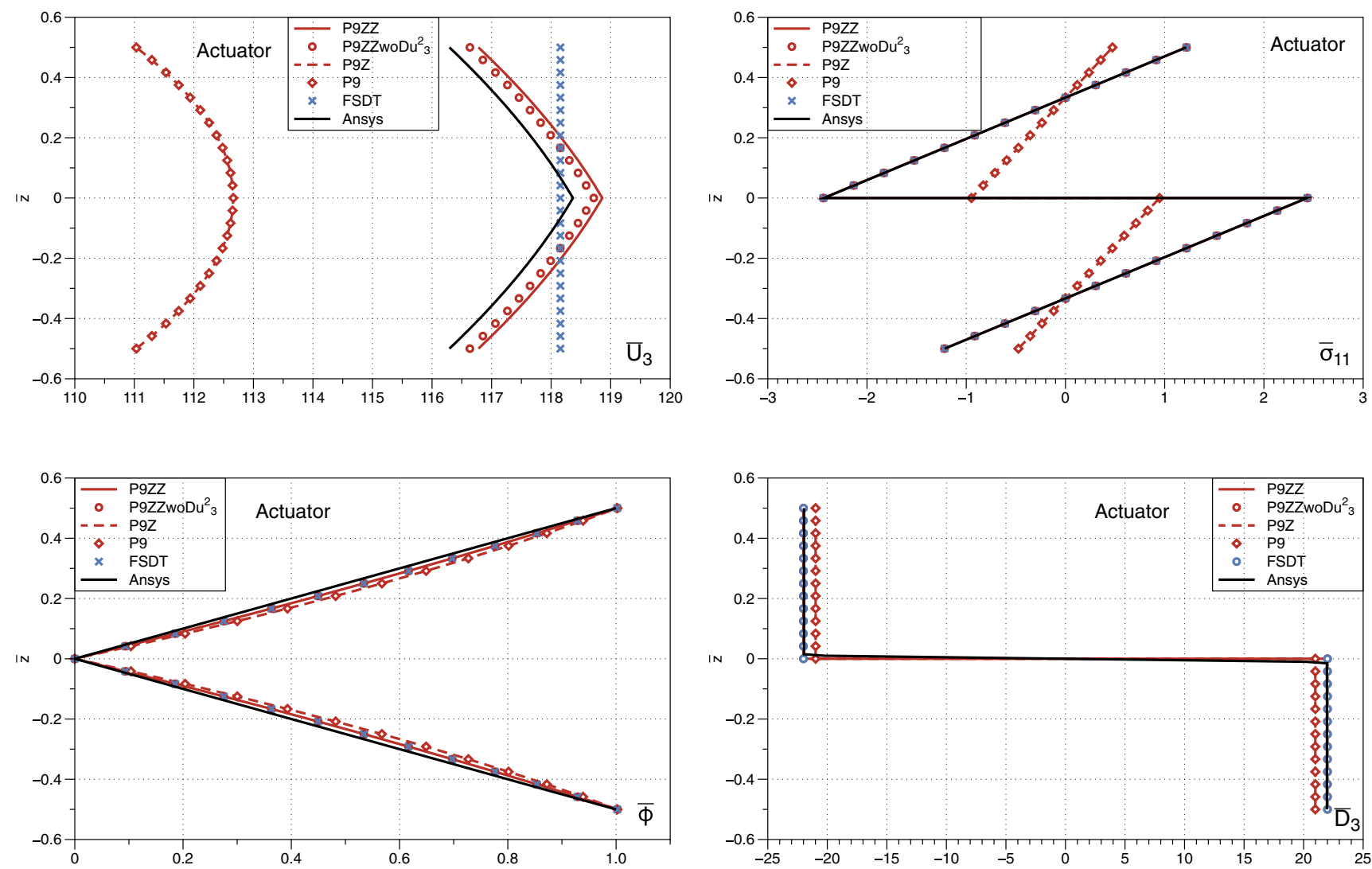

Fig. 2. Parallel bimorph in actuator configuration (cylindrical bending; $S=10 ; 8 \times 2$ mesh): influence of the Zig-Zag terms on the distributions across the thickness.

the same $\Delta \phi$ is imposed on the piezoelectric layer for all elements belonging to the same patch. From the numerical point of view, this is accomplished through linear homogeneous and nonhomogeneous Multi-Point Constraints (MPC) using penalty function method.

In Eq. (16), the load vectors $\left[L_{u}\right]$ and $\left[L_{\phi}\right]$ represent the external loading from applied forces and prescribed charges, respectively. Essential boundary conditions (i.e., prescribed displacements and electric potentials) are imposed numerically by a penalty technique. The coupled system is then solved by the classical static condensation procedure for the electrical dof:

$\left[q_{\phi}\right]=\left[K_{\phi \phi}\right]^{-1}\left(\left[L_{\phi}\right]-\left[K_{u \phi}\right]^{T}\left[q_{u}\right]\right)$

which yields the following purely mechanical system with a modified equivalent stiffness matrix:

$\left[\left[K_{u u}\right]-\left[K_{u \phi}\right]\left[K_{\phi \phi}\right]^{-1}\left[K_{u \phi}\right]^{T}\right]\left[q_{u}\right]=\left[L_{u}\right]-\left[K_{u \phi}\right]\left[K_{\phi \phi}\right]^{-1}\left[L_{\phi}\right]$

In the remainder of the paper, $\left[L_{\phi}\right]=[0]$ will be considered.

\section{Numerical results}

This section is dedicated to the evaluation of the new FEs denoted P9, P9Z and P9ZZ on piezoelectric plate problems. The objectives are pointed towards (i) evaluation of the ZigZag terms influence, regarding the length-to-thickness ratio and the boundary conditions, (ii) introduction of equipotential conditions for modeling not only piezoelectric layers but also piezoelectric patches glued on the bottom or top surface of a composite plate.

\subsection{Parallel bimorph}

The test is presented in Fig. 1 and defined as follows:

Geometry rectangular plate $a \times b$ with $a=25 \mathrm{~mm}$ and $b=12.5 \mathrm{~mm}$; thickness $e$ is defined in order to propose different values for $S=\frac{a}{e}=5,10,50$.

Materials two layers of PZT-4 with parallel poling direction along the $z$-axis; the material properties for PZT- 4 are given in Table 1.

Boundary conditions simply supported at opposite edges $x_{1}=0, a$; two load cases are considered: (i) sensor with uniform pressure load on the top surface $p_{0}(z=e / 2)=1000 \mathrm{Nm}^{-2}$ and $\Delta \phi=0 \mathrm{~V}$ for both layers; (ii) actuator with $\Delta \phi_{0}=50 \mathrm{~V}$ for both layers.

Results and locations for sensor displacements and stresses are made non-dimensional according to

$\left(\bar{U}_{1}, \bar{U}_{3}, \bar{\phi}\right)=\frac{C_{11}}{p_{0} e}\left(U_{1}(0, b / 2, e / 2), U_{3}(a / 2, b / 2,0), \frac{1}{E_{0}} \phi(a / 2, b / 2, e / 4)\right)$

$\left(\bar{\sigma}_{11}, \bar{\sigma}_{13}, \bar{D}_{3}\right)=\frac{1}{p_{0}}\left(\sigma_{11}(a / 2, b / 2, e / 2), \sigma_{13}(a / 4, b / 2,0), E_{0} D_{3}(a / 2, b / 2, e / 2)\right)$.

Results and locations for actuator displacements and stresses are made non-dimensional according to

$$
\begin{aligned}
& \left(\bar{U}_{1}, \bar{U}_{3}, \bar{\phi}\right)=\frac{E_{0}}{\Delta \phi_{0}}\left(U_{1}(0, b / 2, e / 2), U_{3}(a / 2, b / 2,0), \frac{1}{E_{0}} \phi(a / 2, b / 2, e / 4)\right) \\
& \left(\bar{\sigma}_{11}, \bar{\sigma}_{13}, \bar{D}_{3}\right)=\frac{e E_{0}}{C_{11} \Delta \phi_{0}}\left(\sigma_{11}(a / 2, b / 2, e / 2), \sigma_{13}(a / 4, b / 2,0), E_{0} D_{3}(a / 2, b / 2, e / 2)\right) .
\end{aligned}
$$

Jump results at the interface $z=0$ between the PZT- 4 layers, the jump of $\sigma_{11}$ and $D_{3}$ are given using notations $\bar{\sigma}_{11} 0$ and $\left[\left[\bar{D}_{3}\right]\right]_{0}$. 

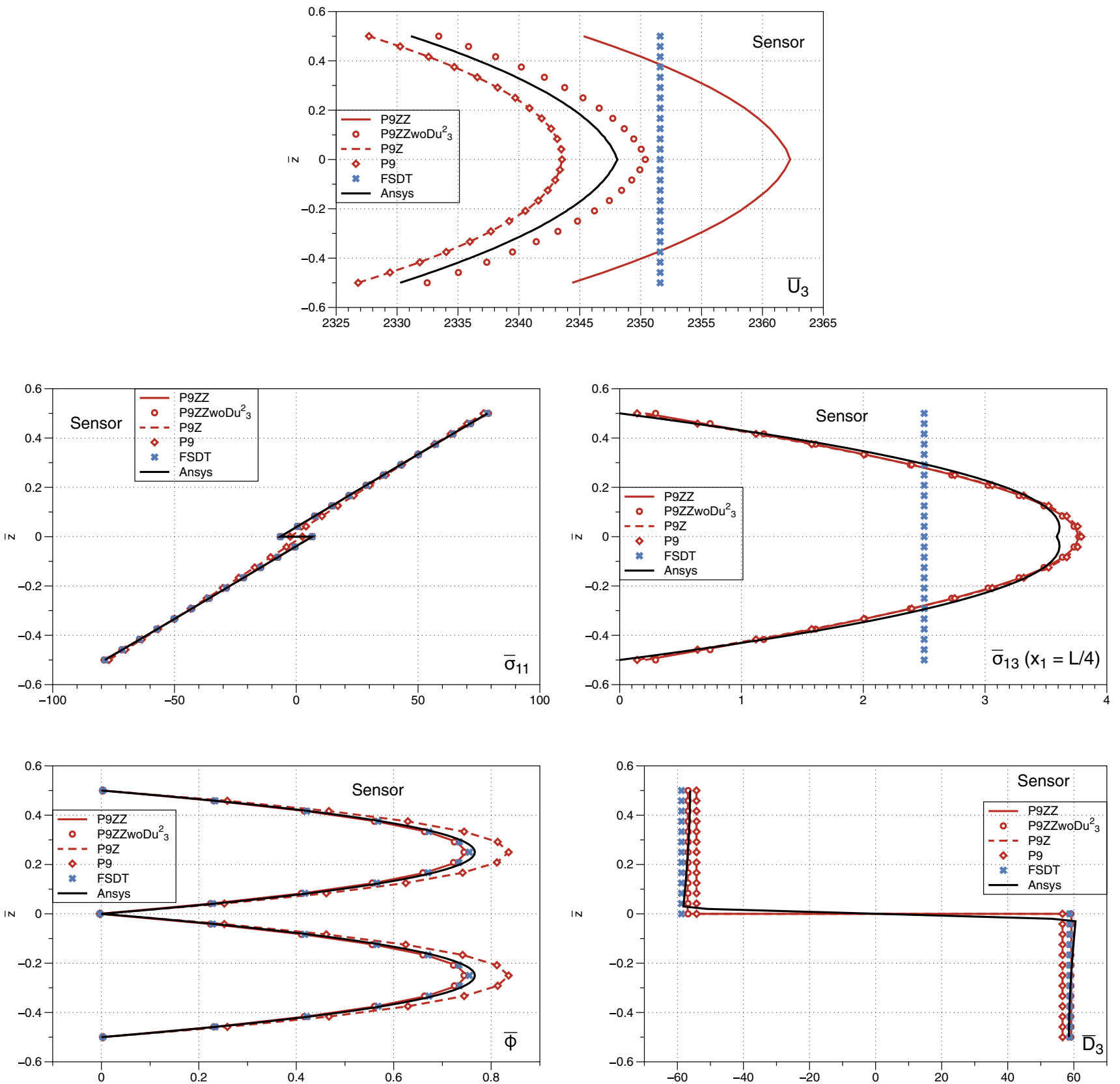

Fig. 3. Parallel bimorph in sensor configuration (cylindrical bending; $S=10 ; 8 \times 2$ mesh): influence of the Zig-Zag terms on the distributions across the thickness.

Table 4

Parallel bimorph in cylindrical bending $(8 \times 2$ mesh): results for different length-to-thickness ratios $S$.

\begin{tabular}{|c|c|c|c|c|c|c|c|c|c|}
\hline$S$ & Model & $\bar{U}_{1}$ & $\bar{U}_{3}$ & $\bar{\sigma}_{11}$ & {$\left[\left[\bar{\sigma}_{11}\right]\right]_{0}$} & $\bar{\sigma}_{13}$ & $\bar{\phi}$ & $\bar{D}_{3}$ & {$\left[\left[\bar{D}_{3}\right]\right]_{0}$} \\
\hline & & Actuator & & & & & & & \\
\hline \multirow[t]{2}{*}{5} & Ansys & -10.63 & 29.94 & 1.21 & 4.87 & 0.0 & 0.50 & -21.96 & 43.92 \\
\hline & P9ZZ & -11.26 & 30.18 & 1.22 & 4.87 & 0.0 & 0.53 & -21.96 & 43.93 \\
\hline \multirow[t]{2}{*}{10} & Ansys & -22.43 & 118.37 & 1.22 & 4.87 & 0.0 & 0.50 & -21.96 & 43.92 \\
\hline & P9ZZ & -23.19 & 118.86 & 1.22 & 4.88 & 0.0 & 0.53 & -22.01 & 44.01 \\
\hline \multirow[t]{3}{*}{50} & Ansys & -116.78 & 2948.0 & 1.22 & 4.87 & 0.0 & 0.50 & -21.96 & 43.92 \\
\hline & P9ZZ & -118.53 & 2967.0 & 1.22 & 4.90 & 0.0 & 0.53 & -22.10 & 44.18 \\
\hline & & bottom/top & Sensor & & & & & & \\
\hline \multirow[t]{2}{*}{5} & Ansys & $46.15 /-44.27$ & 159.66 & 19.83 & 3.40 & 1.79 & 0.198 & -11.94 & 26.70 \\
\hline & P9ZZ & $46.10 /-43.64$ & 166.36 & 19.97 & 2.90 & 1.89 & 0.183 & -12.73 & 27.81 \\
\hline \multirow{2}{*}{10} & Ansys & $366.4 /-362.1$ & 2348.1 & 78.53 & 13.19 & 3.59 & 0.767 & -52.28 & 104.53 \\
\hline & P9ZZ & $367.3 /-362.4$ & 2362.3 & 78.80 & 12.62 & 3.77 & 0.750 & -56.83 & 116.16 \\
\hline \multirow[t]{2}{*}{50} & Ansys & $45.7 \mathrm{E} 3 /-45.6 \mathrm{E} 3$ & $1.43 \mathrm{E} 6$ & 1957. & 326.7 & 17.96 & 18.99 & -1471.0 & 2948.2 \\
\hline & P9ZZ & $46.0 \mathrm{E} 3 /-45.7 \mathrm{E} 3$ & $1.44 \mathrm{E} 6$ & 1966. & 345.1 & 18.68 & 19.03 & -1463.1 & 3132.9 \\
\hline
\end{tabular}


Table 5

Parallel bimorph: cylindrical bending and plate bending models.

\begin{tabular}{|c|c|c|c|c|c|c|c|c|c|}
\hline$S$ & Model & $\bar{U}_{1}$ & $\bar{U}_{3}$ & $\bar{\sigma}_{11}$ & {$\left[\left[\bar{\sigma}_{11}\right]\right]_{0}$} & $\bar{\sigma}_{13}$ & $\bar{\phi}$ & $\bar{D}_{3}$ & {$\left[\left[\bar{D}_{3}\right]\right]_{0}$} \\
\hline \multicolumn{10}{|c|}{ Actuator configuration } \\
\hline \multirow[t]{4}{*}{5} & P9ZZ Cyl. bend. & -11.26 & 30.18 & 1.22 & 4.87 & 0.0 & 0.534 & -21.97 & 43.93 \\
\hline & $\mathrm{F} \& \mathrm{P}[23]$ & - & 27.65 & - & 4.93 & - & - & - & 44.44 \\
\hline & P9ZZ Plate bend. & -8.85 & 24.58 & 1.29 & 5.13 & 0.0 & 0.548 & -23.15 & 46.29 \\
\hline & K\&K [24] & - & 24.01 & - & 5.12 & - & 0.547 & - & 46.15 \\
\hline \multirow[t]{4}{*}{10} & P9ZZ Cyl. bend. & -23.07 & 118.86 & 1.22 & 4.88 & 0.0 & 0.534 & -22.01 & 44.01 \\
\hline & F\&P [23] & - & 116.00 & - & 4.90 & - & - & - & 44.20 \\
\hline & P9ZZ Plate bend. & -23.07 & 97.83 & 1.31 & 5.13 & 0.0 & 0.547 & -23.13 & 46.25 \\
\hline & K\&K [24] & - & 97.68 & - & 5.10 & - & 0.547 & - & 46.05 \\
\hline \multicolumn{10}{|c|}{ Sensor configuration } \\
\hline \multirow[t]{4}{*}{5} & P9ZZ Cyl. bend. & -46.10 & 166.36 & 19.97 & 2.90 & 1.89 & 0.183 & -12.73 & 27.81 \\
\hline & F\&P [23] & - & 161.47 & - & - & 1.86 & 0.206 & - & - \\
\hline & P9ZZ Plate bend. & -49.76 & 181.27 & 19.81 & 2.14 & 1.62 & 0.139 & -9.05 & 21.01 \\
\hline & K\&K [24] & - & 176.10 & - & 2.69 & - & 0.156 & - & 24.28 \\
\hline \multirow[t]{4}{*}{10} & P9ZZ Cyl. bend. & -364.55 & 2362.28 & 78.80 & 12.62 & 3.77 & 0.750 & -56.83 & 116.16 \\
\hline & F\&P [23] & - & 2358.40 & - & - & 3.72 & 0.775 & - & - \\
\hline & P9ZZ Plate bend. & -397.61 & 2579.17 & 77.59 & 9.83 & 3.05 & 0.590 & -44.02 & 91.26 \\
\hline & K\&K [24] & - & 2568.00 & - & 10.58 & - & 0.614 & - & 95.40 \\
\hline
\end{tabular}
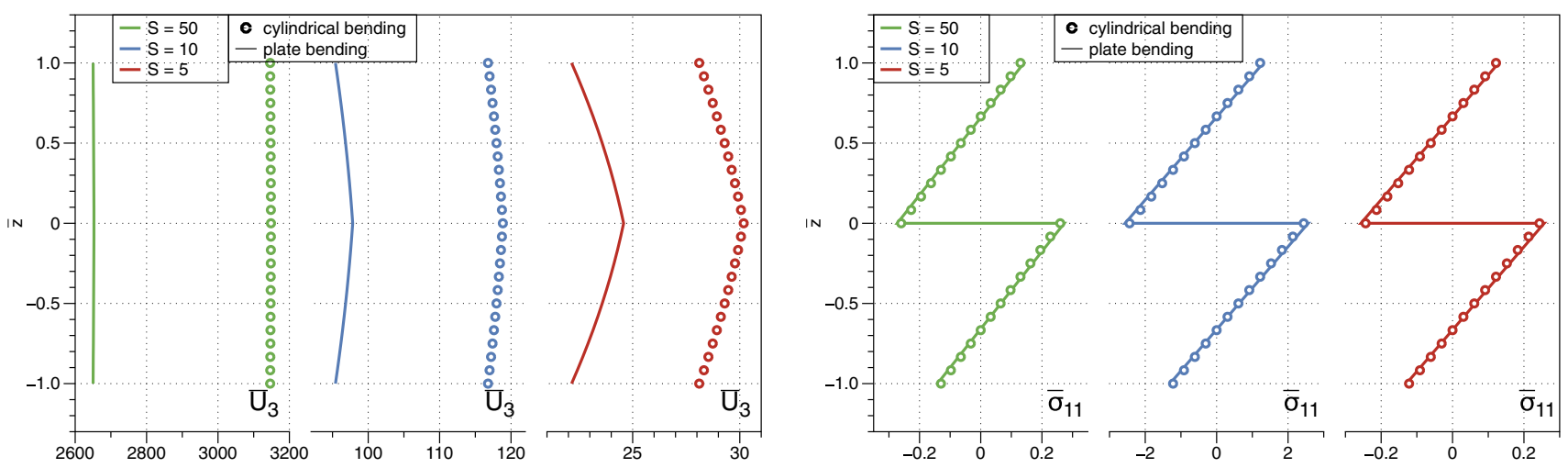

Fig. 4. Cylindrical vs plate bending of parallel bimorph in actuator configuration: through-thickness distributions for three length-to-thickness ratios $S=5,10,50$.

The reference electric field $E_{0}=10^{10} \mathrm{Vm}^{-1}$ is used in the above non-dimensioning coefficients [23].

\subsubsection{Convergence analysis}

A mesh convergence study is first proposed using the FE based on the 12 parameter model with the Zig-Zag functions, denoted P9ZZ. The length to thickness ratio is $S=10$ and the results are presented in Table 2. The number of mechanical and electric dofs is also given. The plate is subjected to a cylindrical bending and the meshes use two elements along the $y$ direction, and all the dofs in this direction are set to zero. The convergence rate is higher for the actuator case than for the sensor one and good agreement is obtained with respect to Ansys results, which refer to a plane strain model with a mesh using $76 \times 46$ finite elements. The error

Table 6

Clamped composite plate with only one electric patch: convergence results for open circuit (OC) and closed circuit (CC).

\begin{tabular}{lllll}
\hline Elec BC & Mesh & $5 \times 2$ & $10 \times 4$ & $20 \times 8$ \\
\hline OC & $\bar{u}_{3}$ & $22.649(-1.9)$ & $22.967(-0.5)$ & 23.089 \\
& $\overline{\Delta \phi}_{\text {bottom }}$ & $-23.003(-2.7)$ & $-23.408(-1.0)$ & -23.649 \\
& $\overline{\Delta \phi}_{\text {top }}$ & $24.473(-2.6)$ & $24.886(-1.0)$ & 25.129 \\
CC & $\bar{u}_{3}$ & $23.370(-1.9)$ & $23.698(-0.3)$ & 23.833 \\
& $\bar{\phi}_{\text {Max }}$ & -3.307 & -5.212 & -7.295 \\
\hline
\end{tabular}

on the maximum values is always less than $1 \%$ except for the electric displacement component which is around $8 \%$. The mesh $8 \times 2$ could be chosen for the subsequent numerical evaluations.

\subsubsection{Influence of the Zig-Zag terms}

The effect of the Zig-Zag terms is assessed in Table 3 for the length to thickness ratio $S=10$, for actuator and sensor configurations. This table presents results obtained using Ansys, different versions of the present high-order model (P9ZZ, P9Z and P9) as well as FSDT. Furthermore, the model (P9ZZ w/o Du $u_{3}^{2}$ ) is deduced from P9ZZ neglecting the contribution of the derivative of $u_{3}^{2}$ in the transverse shear strain, see Eq. (7). The results in Table 3 show that the introduction of the Zig-Zag term in the transverse component of the displacement is necessary to recover results in good agreement with reference solutions for mechanical and electric values. The $\sigma_{11}$ jump at $z=0$ is the most impacted result. It must be emphasized that $\mathrm{P9}$ and $\mathrm{P9Z}$ give the same results, which means that classical Zig-Zag terms acting only in the in-plane displacements do not improve the results for the bimorph structure.

For the actuator configuration, distributions across the thickness are presented in Fig. 2. Introduction of the Zig-Zag term in the transverse displacement $\bar{U}_{3}$ is clearly visible and allows to recover the correct jump of $\bar{\sigma}_{11}$ at $z=0$. This is due to the slope discontinuity of the transverse displacement at layers' interface induced by the Zig-Zag function. On the contrary, P9 and P9Z give 

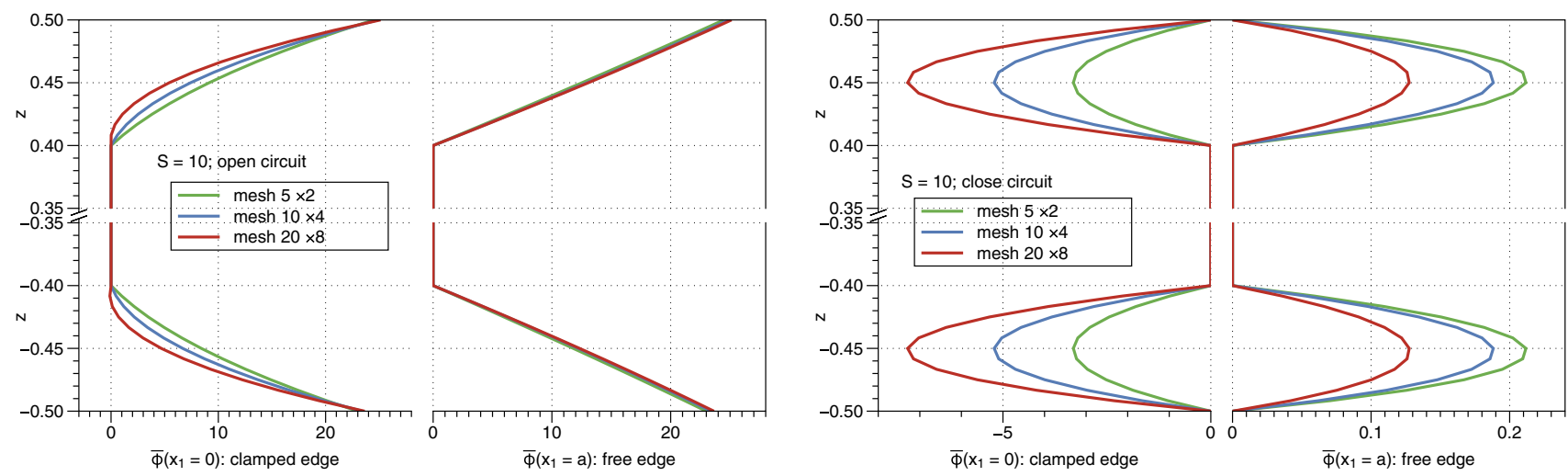

Fig. 5. Clamped composite plate: electric potential distribution across the thickness for the OC (left) and CC (right) electric boundary conditions.

Table 7

Clamped composite plate in OC configuration: evaluations for different FE meshes and equipotential surface sizes.

\begin{tabular}{lllll} 
Patch Nb & 10 & & 40 & 160 \\
Mesh & $5 \times 2$ & $10 \times 4$ & $10 \times 4$ & $20 \times 8$ \\
$\bar{u}_{3}$ & 22.230 & 22.533 & 22.510 & 22.609 \\
$\overline{\Delta \phi}_{\text {bottom }}$ & -62.776 & -63.503 & -80.004 & -91.988 \\
$\overline{\Delta \phi}_{\text {top }}$ & 64.071 & 64.827 & 81.147 & 92.884 \\
\hline
\end{tabular}

a smooth distribution across the thickness for $\bar{U}_{3}$. As expected, FSDT gives a constant distribution for the transverse displacement and accurate distribution for the in-plane stress is obtained thanks to the bi-dimensional constitutive law. All models rely on the same cubic LW approximation for the electric field and the electric response of all models is sensibly the same. Note that the distribution of the electric potential is slightly non-linear across the thickness in each layer.

Fig. 3 presents the sensor configuration results. Transverse displacement distributions from different models are very close to each other, with differences less than $1 \%$, but the change of slope at the layers' interface $(z=0)$ is recovered only by the P9ZZbased models that include the Zig-Zag effect in the transverse direction. For this configuration, all the results are in good agreement with Ansys reference solutions.

\subsubsection{Results for varying length-to-thickness ratios}

The influence of the length-to-thickness ratio on the parallel bimorph configuration is investigated in Table 4 , where the results for thin to very thick plates obtained by the P9ZZ model are compared against those obtained by Ansys. A very good accuracy is recovered for displacement, in-plane and transverse shear stresses, and electric potential, for both actuator and sensor configurations. The maximum error is found for the jump of normal electric displacement in the sensor configuration: for $S=5,10$ and 50 the error is 4,11 and $6 \%$, respectively. Note that for the sensor configuration, in-plane displacements at bottom and top surfaces are very different for thick and semi-thick ratios and tend towards the same value in the thin plate range $S=50$, i.e., the symmetry with respect to $z=0$ is recovered when the plate becomes thin.

\subsubsection{Plate bending vs cylindrical bending of bimorph}

A last numerical study is proposed for the bimorph structure by comparing the cylindrical bending approach considered so far with the classical plate bending. Results are obtained using an $8 \times 2$ mesh for cylindrical bending and an $8 \times 8$ mesh for plate bending. The results for actuator and sensor configurations and two lengthto-thickness ratios $(S=5,10)$ are presented in Table 5. Results from Fernandes and Pouget [23] and Kapuria and Kulkarni [24] are added for comparison purpose, when they are available. In this last paper, the value $\sigma_{11}(z=0)$ is reported instead of $\left[\left[\sigma_{11}\right]\right]_{0}$; the value indicated in Table 5 has been computed by multiplying the reported value by 2 . It is obvious that Kapuria and Kulkarni [24] consider plate bending configuration while Fernandes and Pouget [23] deal with a plane strain model (cylindrical bending). Note that in the sensor case, displacements for cylindrical bending are lower than for plate bending, whereas the opposite holds true for the actuator case. In all cases, P9ZZ results are in good agreement with
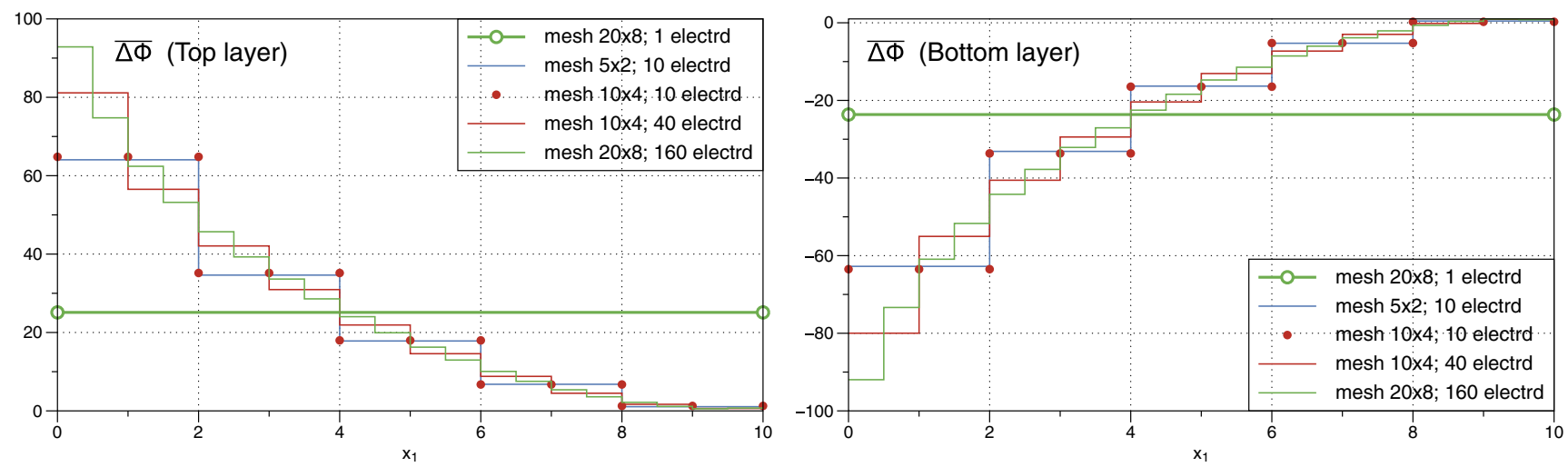

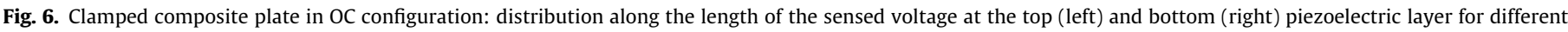
meshes and electrode segmentation. 
the different reported results. Finally, Fig. 4 presents for the actuator configuration the distributions across the thickness of $u_{3}$ and $\sigma_{11}$ for different length-to-thickness ratios: the cylindrical and plate bending cases yield similar distributions but the numerical values are larger for the former case.

\subsection{Clamped composite plate}

This test is interesting to evaluate the segmentation of a piezoelectric layer using different number of patches. It considers the sensory response of a cantilever, rectangular, hybrid sandwich plate with one edge clamped and other edges free subjected to a uniform pressure load according to the following data [24]:

Geometry rectangular plate $a \times b$ with $a=10 \mathrm{~mm}, a / b=2$ and total thickness $e=1 \mathrm{~mm}$ (length-to-thickness ratio $S=10$ ).

Materials seven-layers plate $(p z, \angle 0, \angle 90$, core, $\angle 90, \angle 0, p z)$; the outer piezoelectric layers of thickness $0.1 \mathrm{e}$ are made out of PZT-5A material; each layer constituting the laminated skins has a thickness $0.04 e$ and the core thickness equals $0.64 e$. The material properties for PZT-5A, skin layers and core are given in Table 1.

Boundary conditions clamped at $x_{1}=0$; uniform pressure load $p_{0}$; piezoelectric layers can be in open circuit ( $\Delta \phi$ let free) or in closed circuit $(\Delta \phi=0)$.

Mesh three regular meshes $5 \times 2,10 \times 4$ and $20 \times 8$ are used.

Results and locations transverse displacement at the tip and piezoelectric potential in the element close to the clamped edge. They are made non-dimensional according to

$$
\begin{aligned}
& \bar{u}_{3}=u_{3}\left(10^{2} E_{2}^{(\text {core })}\right) /\left(e S^{4} p_{0}\right) \\
& \bar{\phi}=\phi\left(10^{4} E_{2}^{(\text {core })} d_{0}\right) /\left(e S^{2} p_{0}\right)
\end{aligned} \text { with } d_{0}=37410^{-12} \mathrm{CN}^{-1} .
$$

This test can be used to compare the effect of the electrode segmentation: using the proposed FE meshes, the electrode sensor surface is subdivided in $1,10,40,160$ patches through the imposition of equipotential conditions.

Table 6 presents the convergence properties of the P9 FE when only one patch is used for each piezoelectric layer. In the open circuit configuration, the equipotential condition enforces $\Delta \phi$ in each layer to be the same for all elements. In the closed circuit condition, $\Delta \phi=0$ for all elements (equipotential condition) and the maximum electric potential value inside the piezoelectric layers is reported. Note that the cubic approximation for $\phi$ allows to recover the electric potential induced by the local bending of the piezoelectric layers.

The maximum displacement is located at the center of the tip edge $(a, b / 2)$ while the maximum induced electric potential $\left(\phi_{\max }\right.$ for closed circuit) is in the FE close to the clamped edge. For the open circuit, the convergence is very fast for both displacement and electric potential difference - the percentage of error with respect to the $20 \times 8$ mesh results is indicated in parentheses. For the closed circuit, an asymptotic value is recovered for the displacement whereas $\phi_{\max }$ still increases with mesh refinement; this is due to the decreasing size of the FE at the clamp, which increasingly localizes the bending deformation of the piezoelectric layers.

The distribution of the electric potential across the thickness is illustrated for the two electric boundary conditions in Fig. 5. The non-linear variation across the bottom and top piezoelectric layers is visible on both graphs and increases with mesh refinement. Due to the equipotential condition, it has no influence on the $\Delta \Phi_{\max }$ for the open circuit (left) but it is directly related to the $\Phi_{\max }$ for the closed circuit (right). This non-linear effect measures the local bending deformation of the piezoelectric layers and is accordingly larger at the clamped edge than at the free tip of the plate.

Finally, the effect of electrode segmentation is discussed in Table 7 for the open circuit boundary condition. The corresponding distribution along the length of the plate of the electric potential difference is illustrated in Fig. 6 for all considered meshes and electrode segmentations. The induced potential difference $\Delta \phi$ reported in Table 7 is taken at the electrode next to the clamped edge, i.e., where it has its maximum value. Comparing the results for 10 patches obtained with two different meshes allows to appreciate the role of the mesh refinement: a finer mesh increases the tip displacement and enhances the sensed voltage. The same mesh with $10 \times 4$ elements is then used with two different number of electric patches, i.e., with different sizes of the equipotential surfaces: a very small influence on the tip deflection can be seen, with $\bar{u}_{3}$ slightly larger when the equipotential surfaces are larger; on the contrary, the electric potential induced at the clamped edge is clearly higher the smaller the electrode. Finally, considering the $20 \times 8$ with 160 patches (i.e., one electrode per FE), the tip displacement is slightly higher due to the refined mesh and the sensed voltage is further increased due to both, the larger deflection and the reduced electrode's size at the clamp.

\section{Conclusion}

This paper has presented a new family of FE for piezoelectric composite plates. A high-order ESL kinematic model is considered that includes the sinus function for the in-plane displacements, a quadratic polynomial expansion for the transverse displacement and Zig-Zag functions for introducing slope discontinuities at layers' interfaces for both, the in-plane and the transverse displacements. A LW cubic approximation is used for the electric potential in order to capture its non-linear distribution induced by the local bending of the piezoelectric layer; the elementary domain is considered as an equipotential surface (constant electric potential).

The element has been validated through linear static case studies for both sensor and actuator configurations as well as homogeneous and laminated plates. New reference solutions have been provided by means of 2D FEM computations with a commercial software. It turned out that the Zig-Zag term along the transverse direction is essential for recovering accurate results when dealing with piezoelectric bimorph structures. The role of electrode segmentation, i.e., the size of equipotential surfaces, on the electromechanical response has been also evaluated. The results are in good agreement for actuator and sensor configurations for thin to very thick cases. The proposed P9ZZ FE, using only 12 mechanical dof per node, is very accurate, simple to use, without any numerical problem and could be used for a large range of plate problems involving piezoelectric patches or layers. Future works are pointed towards the extension of this model to piezoelectric shell structures.

\section{References}

[1] Chopra I. Review of state of art of smart structures and integrated systems. AIAA J 2002;40:2145-87.

[2] Polit O, Bruant I. Electric potential approximations for an eight node plate finite element. Comput Struct 2006;84:1480-93.

[3] Gopinathan S, Varadan V, Varadan V. A review and critique of theories for piezoelectric laminates. Smart Mater Struct 2000;9(1):24-48.

[4] Gibson R. A review of recent research on mechanics of multifunctional composite materials and structures. Compos Struct 2010;92:2793-810.

[5] Carrera E, Boscolo M, Robaldo A. Hierarchic multilayered plate elements for coupled multifield problems of piezo-electric adaptive structures: formulation and numerical assessment. Arch Comput Methods Eng 2007;14(4):383-430.

[6] Vidal P, D'Ottavio M, Ben Thaïer M, Polit O. An efficient shell finite element for the static response of piezoelectric laminates. J Intell Mater Syst Struct 2011;22(7):671-90. 
[7] Murakami H. Laminated composite plate theory with improved in-plane response. J Appl Mech 1986;53:661-6.

[8] Polit O, Vidal P, D’Ottavio M. Robust $c^{0}$ high-order plate finite element for thin to very thick structures: mechanical and thermo-mecanical analysis. Int J Numer Methods Eng 2012;90(4):429-51.

[9] Jiang J, Li D. A new finite element model for piezothermoelastic composite beam. J Sound Vib 2007;306:849-64.

[10] Beheshti-Aval SB, Lezgy-Nazargah M, Vidal P, Polit O. A refined sinus finite element model for the analysis of piezoelectric-laminated beams. J Intell Mater Syst Struct 2011;22(3):203-19.

[11] A.S. 176. IEEE standard on piezoelectricity, American National Standard Institute; 1987.

[12] Tiersen H. Linear piezoelectric plate vibrations. New-York: Plenum; 1969.

[13] Touratier M. An efficient standard plate theory. Int J Eng Sci 1991;29:901-16.

[14] Touratier M. A refined theory of laminated shallow shells. Int J Solids Struct 1992;29(11):1401-15.

[15] Touratier M. A generalization of shear deformation theories for axisymmetric multilayered shells. Int J Solids Struct 1992;29:1379-99.

[16] Ganapathi M, Patel B, Polit O, Touratier M. A $c^{1}$ finite element including transverse shear and torsion warping for rectangular sandwich beams. Int J Numer Methods Eng 1999;45(1):47-75.
[17] Polit O, Touratier M. A multilayered/sandwich triangular finite element applied to linear and nonlinear analysis. Compos Struct 2002;58(1):121-8.

[18] Dau F, Polit O, Touratier M. $C^{1}$ plate and shell finite elements for geometrically non linear analysis of multilayered structures. Comput Struct 2006;84:1264-74.

[19] Vidal P, Polit O. A thermomechanical finite element for the analysis of rectangular sandwich beams. Finite Elem Anal Des 2006;42:868-83.

[20] Vidal P, Polit O. A family of sinus finite elements for the analysis of rectangular laminated beams. Compos Struct 2008;84:56-72.

[21] Cheng S. Elasticity theory of plates and a refined theory. J Appl Mech ASME 1979;46:644-50.

[22] Polit O, Touratier M. High order triangular sandwich plate finite element for linear and nonlinear analyses. Comput Methods Appl Mech Eng 2000; $185: 305-24$.

[23] Fernandes A, Pouget J. Analytical and numerical approaches to piezoelectric bimorph. Int J Solids Struct 2003;40:4331-52.

[24] Kapuria S, Kulkarni S. Static electromechanical response of smart composite sandwich plates using an efficient finite element with physical and electric nodes. Int J Mech Sci 2009;51:1-20. 